\title{
The Impact of the Falsified Medicines Directive in the Dispensing Operations of an Austrian Hospital Pharmacy
}

\begin{abstract}
The Falsified Medicines Directive (FMD) and the Delegated Regulation (DR) will need to be implemented in the next couple of years to ensure compliance of dispensing operations in the secondary environment. This study was developed in an Austrian hospital, using a medicines authentication system to assess the impact of the implementation of the legal requirements of the FMD. The orders were dispensed using current daily procedures where the operational dispensing time was assessed and compared with those processed through an authentication system. The conclusions of this study confirm that the regulatory implementation of the FMD to hospital dispensing procedures will increase the operational dispensing time.
\end{abstract}

Keywords: Falsified medicines directive; Hospital pharmacy; Health care

Received: March 14, 2017; Accepted: March 30, 2017; Published: April 05, 2017

\section{Introduction}

The prevalence of counterfeit medicines has risen internationally in recent years [1]. According to the Pharmaceutical Security Institute, there were 2077 incidents in 2014, up from 196 in 2002 [2]. This increase demonstrates an emerging healthcare problem to societies. The increased incidence can be due to the improved security and detection methods of national competent authorities or the genuine increase of falsified medicines in the supply chain.

Several European Union member states in the last decade have legislated on this issue. There is a range of legislation relating to falsified medicines in Europe. Such legislation includes the Bollini law introduced in Italy in 2000, which requires medicines to be tracked to the point of sale using two barcodes and laws in Belgium and Greece, introduced in 2005, which have resulted in mass serialisation of medicines by manufacturers $[3,4]$. The purpose of introducing serialisation was to assist with the reimbursement of medicines by the department of health of the respective countries. The Falsified Medicines Directive (FMD) was introduced in 2011 by the European Union, and aims to harmonise legislation across Europe and to ensure the highest standards in medicines authentication.

By 2019 , all member states must be fully compliant with the regulations set out in the FMD Delegated Regulation (DR). The DR was published in February 2016. Implementation of this directive will be completed in 3 years, from the date of the publication, and requires significant adaptation of the pharmaceutical
Pinto de $\mathrm{CN}^{1,2}$, Koshkouei $\mathrm{MJ}^{3}$, Jeske $\mathrm{M}^{4}$, Zeiler $\mathrm{M}^{4}$ and Brindley $\mathrm{DA}^{3,5-8}$

1 Aegate Ltd, H9 the Courtyard, Melbourn Science Park, Herts., UK

2 Open University, Business School, Walton Hall, Milton Keynes, UK

3 Harvard Stem Cell Institute, Cambridge, MA, 02138, USA

4 Pharmacy Department, Innsbruck University Hospital, Innsbruck, Austria

5 The Oxford - UCL Centre for the Advancement of Sustainable Medical Innovation (CASMI), The University of Oxford, New Richards Building, Old Road Campus, Oxford, OX3 7LG, UK

6 Department of Paediatrics, University of Oxford, Level 2, Children's Hospital, John Radcliffe Hospital, Oxford, OX3 9DU, UK

7 Centre for Behavioural Medicine, UCL School of Pharmacy, University College London, BMA House, Tavistock Square, London WC1H 9JP, UK

8 USCF-Stanford Centre of Excellence in Regulatory Science and Innovation (CERSI), USA

Corresponding author: Pinto de Castro N

= nuno.pintodecastro@gmail.com

Aegate Ltd, H9 the Courtyard, Melbourn Science Park, Herts, UK.

Tel: +44 1763268160

Citation: de CNP, Koshkouei MJ, Jeske M, et al. The Impact of the Falsified Medicines Directive in the Dispensing Operations of an Austrian Hospital Pharmacy. J Hosp Med Manage. 2017, 3:1.

supply chain before the 2019 deadline [5,6]. The National Medicines Verification Organisations (NMVO) is responsible for implementing the National Medicines Verification System (NMVS) in each member state of the EU [7]. 
The lack of understanding of the potential impact on hospital authentication of the implementation of a medicines authentication system (MAS) in the secondary care environment is evident.

The introduction of MAS is likely to result in a large upheaval for secondary care pharmacy staff if not managed appropriately. It is of vital importance to validate the medicines authentication system in preparation for the 2019 deadline in order to facilitate a fluid transition. It is critical to optimise the dispensing process and establish best practice for medicine authentication in secondary care before the 2019 deadline $[5,6]$.

\section{FMD and the impact for dispensing activities in the hospital pharmacy}

To better understand the impact of the implementation of MAS in the hospital pharmacy environment, a one-month project was performed in the hospital pharmacy of Innsbruck University Hospital. The study was developed in July 2016, as an interventional study. A maximum of four orders received in the hospital pharmacy each day for the entire month were tested trough the authentication system and the normal dispensing operations by the same staff member. A comparison between the operational dispensing time (ODT) between the current operation and in a FMD environment, through the authentication system, was undertaken to understand the impact of the implementation of the FMD in the secondary care environment and to identify methods for supply chain optimisation.

Decommission was performed in the dispensary before supplying the products to the wards or other hospitals, as this is the location with the lowest operational time. The members involved in the study that manipulated, dispensed and checked medicines adapted the actual operations to a FMD environment.

Under the DR, two different processes can be distinguished: Verification and Decommissioning. Verification and decommission are different dispensing operations through an authentication system. The medicinal product can be verified, via the 2D bar code matrix, with the respective serial number queried in a future national database and it can therefore be determined whether the scanned number is stored and if the product is genuine. Verification can be performed when it is required. The decommission scanning of the unique identifier of a medicinal product removes the serial code from the national database. This confirms that the product has been dispensed [7].

The system doesn't provide auditability of who the users have been, which would be desirable to access who performs the authentication. It was not possible to directly link individual scans to individual named participants or even patients, confidentiality was therefore assured.

\section{Authentication System and Project Design}

The study was performed using an authentication software system and hardware provided by Aegate Ltd., an authentication company. A standalone laptop and scanner were provided for the project. To simulate a national repository system, a database was generated, containing information about certain medicines which are frequently dispensed or classified as high cost. When one of these medicines was scanned, the status of the unique identifier was updated, the identification of the product was confirmed and a feedback alert was generated. During the project, the average response time was also tested.

\section{Innsbruck University Hospital}

The study was developed in the Tirol Kliniken $\mathrm{GmbH}$, based in Innsbruck, a health care institution which has been operational since 1990.

The Innsbruck University Hospital pharmacy supplies nearly all regional hospitals in the Austrian Tirol, except the Schwaz and Lienz hospitals, a total of 16 hospitals, thereby acting as a centralised hub for the region. Orders are received in the Tirol Kliniken $\mathrm{GmbH}$ from the different hospitals in the region, items are dispensed in the great majority by bulk and distribute daily to hospitals in the region. The hospitals that are supplied do not have a hospital pharmacy.

The central hospital pharmacy in the region dispenses a total of 2.25 million packages a year. The main hospital is the University Hospital of Innsbruck that receives 1.14 million packages of the 2.25 million packages dispensed. It Is the central hospital of the Tirol region of Austria.

Per year a total of 112.000 orders are received and dispensed for the entire group of hospitals.

The group of hospitals has a capacity of 4898 beds; in terms of logistics the implication of the FMD for this type of operation will be significant.

\section{Average response time}

The output of the results includes data entered into the authentication system, data retrieved by authentication technology and data detected by staff.

The average response time through the authentication system was: $366 \mathrm{~ms}$.

The DR states that the response time of the national repository when performing verification or decommission needs to be under 300 milliseconds, not considering the internet connection (Article 35 (f)) [7]. The results achieved were close to that figure, with wireless connection affecting the overall performance. The hardware was not connected to a LAN solution, so the wireless connectivity made a significant difference. Furthermore, the repository system was based in the UK and the response time could be delayed through the connectivity between Austria and the UK.

Nevertheless, the chronostasis perception of a waiting time is above $500 \mathrm{~ms}$. The result achieved did not interfere with the dispensing operation or the operator's awareness of the increased waiting time $[8,9]$. 
So, internet connectivity and the location of the national repository can affect the authentication system and should be considered by the hospitals and NMVO respectively.

\section{Current operational dispensing time vs. operational dispensing time in a FMD environment}

A total of 80 orders were dispensed through the actual dispensing procedures and the exact same order was dispensed through the authentication system for the duration of this study.

In terms of drawing a comparison between the dispensing operations today and in a FMD environment the following results were obtained (Table 1).

There is an increase of the operational dispensing time in a FMD environment. The results were compared between the operational time effectuated in the current daily dispensing operation and in a FMD compliant authentication system. The main reason for this significant change is due to the obligatory scanning of each bar code of a product. The DR states that a product needs to be decommissioned before supplied to the public (Article 25).

At present the Innsbruck hospital pharmacy scans a code for a specific product just for inventory management purposes. If an order is required for 100 packages of the same medicinal product there is only a need to scan one of the bar codes and there is an input of the total amount that is going to be dispensed, this will need to change with the FMD. Each of the packages will have a single unique identifier that needs to be decommissioned and removed from the national repository system when it is dispensed. So the staffs in that specific order will need to scans the 100 packages. This will increase the workload for the hospital pharmacy, an $81 \%$ increase of the operational dispensing time was observed.

For the total orders completed in a year, the hospital pharmacy under the FMD requirements will need to increase to 3.5 full time employees to dispense the 112.000 orders by extrapolating the operational dispensing time achieved in this study, assuming that the same amount of orders are going to be dispensed in Innsbruck hospital in the future (Table 2).

Due to the Innsbruck hospital pharmacy being a centralised dispensing hub for other hospitals in the region, an analysis was made between the orders dispensed for the Innsbruck Hospital and for the other hospitals in the region.

An increase of $47 \%$ of the operational dispensing time was observed for the medicinal products dispensed for the Innsbruck hospital but an increase of $157 \%$ was achieved for products dispensed for other hospitals. It is clear that hospitals that operate this way will have a higher operational dispensing time for products dispensed for other institutions as their operation is similar to that of a wholesaler. The products are dispensed by bulk and normally are requested in large quantities. Orders that have a higher quantity of products will have a greater impact in the
Table 1: Operational dispensing time between a normal dispensing scenario and an FMD environment; ODT - Operational dispensing time; FTE - Full time employee.

\begin{tabular}{|c|c|c|}
\hline 80 Orders & $\begin{array}{c}\text { Normal dispensing } \\
\text { operations }\end{array}$ & FMD Environment \\
\hline ODT (min) & 337 & 608 \\
\hline ODT (hour) & 5.62 & 10 \\
\hline ODT (h/year) & 12298 & 21960 \\
\hline (h/day) & 33.7 & 60 \\
\hline FTE per 365 days & 4.21 & 7.5 \\
\hline
\end{tabular}

Table 2: Operational dispensing time between a normal dispensing scenario and an FMD environment to different Innsbruck hospital and external hospitals; ODT - Operational dispensing time.

\begin{tabular}{|c|c|c|}
\hline & $\begin{array}{c}\text { Orders dispensed to } \\
\text { Innsbruck University } \\
\text { hospital }\end{array}$ & $\begin{array}{c}\text { Orders dispensed to } \\
\text { other hospitals }\end{array}$ \\
\hline ODT MobLink (s) & 14085 & 6139 \\
\hline ODT MobLink (min) & 235 & 102 \\
\hline ODT Aegate (s) & 20737 & 15793 \\
\hline ODT Aegate (min) & 345 & 263 \\
\hline Increase ODT (\%) & 47 & 157 \\
\hline
\end{tabular}

operational dispensing time due to the regulatory requirements of scanning each package that is dispensed.

\section{Automation or semi-automatic solutions can reduce the impact of the FMD?}

Automation has been increasing in the hospital pharmacy environment since the 1960's. Solutions can be installed in the hospital pharmacy to aid the implementation of the FMD and reduce its impact. Nevertheless, with the recent government funding cuts and demand for more efficiency of healthcare systems in Europe the installation of such products can be difficult [10] (Table 3).

Automation can reduce the burden of implementation of the FMD. There are systems in place that can dispense 2000-2400 packages per hour. The Innsbruck hospital pharmacy dispenses 2.25 million packages a year. A total of 1125 hours will be sufficient through robotic automation dispensing to dispense the total number of packages in a year.

A conveyor belt with image recognition system or robotic dispensing of packaging will need to operate approximately 4-5 hours a day to achieve the number of packages dispensed in a year, significantly reducing the increase in staff required in an FMD environment. Part of the dispensing staff can be allocated to other activities such as clinical pharmacy, cytotoxic manipulation or management and procurement improving the pharmaceutical services done in the hospital.

The implementation of such solutions can be extended to a full daily shift if needed and can ultimately be enough to allocate the necessary resources to the dispensing operations. 
Table 3: Performance of different automation solution for the hospital pharmacy environment.

\begin{tabular}{|c|c|c|}
\hline & $\begin{array}{c}\text { Package dispensing } \\
\text { robot Performance }\end{array}$ & $\begin{array}{c}\text { Number of packages } \\
\text { dispensed }\end{array}$ \\
\hline $\begin{array}{c}\text { Package dispensing } \\
\text { robot performance }\end{array}$ & Dispense (hour) & 2000 \\
\hline $\begin{array}{c}\text { Conveyor belt } \\
\text { dispensing performance }\end{array}$ & Dispense (s) & $\sim 2$ \\
\hline & Dispense (s) & 2400 \\
\hline
\end{tabular}

\section{Conclusions}

During this study it was noticed that the implementation of FMD in the hospital pharmacy can generate an impact to dispensing operations. Compared with the community pharmacy, a much greater degree of planning and organization is needed to cope with the decommissioning of large number of medicine packs.

A possible increase in expenditure for the hospital pharmacy due to the need to have more employees can be a reality, so optimisation of the workflow and supply chain needs to be implemented to minimise the impact of the FMD in operations. The DR also introduces the concept of code aggregation that could reduce the impact of dispensing operations but that is a decision for the pharmaceutical industry to consider.

Automation can be a possible solution for hospitals that dispense large quantities of medicines each year. The data collected during the study relates to a period of one month and only four orders were selected per day to reduce the impact on the daily operations of the hospital pharmacy. In order to make more representative statements an extended study period is required. Also an important aspect to be studied in the future are the out of hours' processes, such has weekends that can be impacted by the implementation of the FMD.

\section{Disclosures}

The content outlined herein represents the individual opinions of the authors and may not necessarily represent the viewpoints of their employers. D.A.B and N.P.C. are employees and/or stockholders in Aegate Ltd. (Melbourn, UK) that is a provider of medicines authentication services.

N.P.C is a registered Pharmacist with the General Pharmaceutical Council of Great Britain, the Royal Pharmaceutical Society and the Portuguese Pharmaceutical Society and is bound by the codes of practice and ethics. D.A.B. is also a stockholder in Translation Ventures Ltd. (Charlbury, Oxfordshire, UK) and IP Asset Ventures (Oxford, UK). D.A.B. is subject to the CFA Institute's Codes, Standards, and Guidelines, and as such, this author must stress that this piece is provided for academic interest only and must not be construed in any way as an investment recommendation. Additionally, at time of publication, D.A.B. and the organizations with which he is affiliated may or may not have agreed and/or pending funding commitments from the organizations named herein. M.J.K. is a registered Pharmacist with the General Pharmaceutical Council of Great Britain and is bound by the codes of practice and ethics. M.J.K. is in receipt of research funding from Aegate Ltd. 


\section{References}

1 Dajani S (2013) Falsified Medicines Cross-Board working group, RPS Policy Statement: Falsified medicines directive.

2 PSI-Inc (2015) Pharmaceutical Security institute.

3 Missoni E,Tediosi F,Pacileo G,Gautier L (2014) Italy's Contribution to Global Health: The Need for a Paradigm Shift. Globalization and Health 10: 25.

4 Naughton BD, Smith JA, Brindley DA (2015) Establishing Good Authentication Practise in Secondary Care to protect Against Falsified Medicines and Improve Patient Safety. European Journal Of Hospital Pharmacy 23.

5 European Union (2011) Directive 2011/62/Eu of the European Parliament and of the Council of 8 June 2011 amending directive $2001 / 83 /$ Ec on the community code relating to medicinal products for human use, as regards the prevention of the entry into the legal supply chain of falsified medicinal products. Official journal of the European union, pp: 74-87.
6 Smith JA, Naughton B, Kramm A, Smith G, Simone MD et al European Union Falsified Medicines Directive: Requirements and Implications for Multi-Stakeholder Healthcare Delivery. Regulatory Affairs Professionals Society.

7 Commission Delegated Regulation (EU) 2016/161 of 2 October 2015 supplementing Directive 2001/83/EC of the European Parliament and of the Council by laying down detailed rules for the safety features appearing on the packaging of medicinal products for human use.

8 Hodinott Hill, Thilo KV, Cowey A, Walsh V (2002) Auditory Chronostasis: Hanging on the Telephone. 12: 1779-81.

9 Yarrow K, Whiteley L, Rothwe JC, Haggard P (2006) Spatial consequences of bridging the saccadic gap 46: 545-555.

10 Fitzpatrick R, Cooke P, Southall C, Kauldhar K (2005) Waters $P$ evaluation of an automated dispensing system in a hospital pharmacy dispensary. 